\title{
Global Bounded Solutions for the Keller-Segel Chemotaxis System with Singular Sensitivity
}

\author{
Khalid Ahmed Abbakar1,2*, Omer Khalil1', Alhussein Mohamed1, Bechir Mahamat1, \\ Abdoulaye Ali ${ }^{1}$, Abeer Alhadi ${ }^{3}$
}

${ }^{1}$ College of Mathematics and Statistics, Northwest Normal University, Lanzhou, China

${ }^{2}$ Department of Mathematics and Physics, Faculty of Education, University of Gadarif, Gadarif, Sudan

${ }^{3}$ College of Physics and Electronic Engineering, Northwest Normal University, Lanzhou, China

Email: *khalidahmed200@hotmail.com

How to cite this paper: Abbakar, K.A., Khalil, O., Mohamed, A., Mahamat, B., Ali, A. and Alhadi, A. (2020) Global Bounded Solutions for the Keller-Segel Chemotaxis System with Singular Sensitivity. Applied Mathematics, 11, 985-990.

https://doi.org/10.4236/am.2020.1110064

Received: September 17, 2020

Accepted: October 16, 2020

Published: October 19, 2020

Copyright $\odot 2020$ by author(s) and Scientific Research Publishing Inc. This work is licensed under the Creative Commons Attribution International License (CC BY 4.0).

http://creativecommons.org/licenses/by/4.0/

\section{Abstract}

In this paper, we consider the Neumann initial-boundary value problem for the Keller-Segel chemotaxis system with singular sensitivity

$$
\left\{\begin{array}{l}
u_{t}=d_{1} \Delta u-\chi \nabla \cdot\left(\frac{u}{v} \nabla v\right), \\
v_{t}=d_{2} \Delta v-v+u
\end{array}\right.
$$

is considered in a bounded domain with smooth boundary, $\Omega \subset \mathbb{R}^{n}(n \geq 1)$, where $d_{1}>0, d_{2}>0$ with parameter $\chi \in \mathbb{R}$. When $d_{1}=d_{2}+\chi$, satisfying for all initial data $0 \leq u_{0} \in C^{0}(\bar{\Omega})$ and $0<v_{0} \in W^{1, \infty}(\Omega)$, we prove that the problem possesses a unique global classical solution which is uniformly bounded in $\Omega \times(0, \infty)$.

\section{Keywords}

Keller-Segel System, Chemotaxis, Global Bounded Solution, Singular Sensitivity

\section{Introduction}

The Keller-Segel system is used to model chemotactic movement in biology [1]. The mathematical study of the system has attracted great interest in recent years [2]. In this paper, we consider the Neumann initial-boundary value problem for the chemotaxis system with singular sensitivity 


$$
\begin{cases}u_{t}=d_{1} \Delta u-\chi \nabla \cdot\left(\frac{u}{v} \nabla v\right), & x \in \Omega, t>0 \\ v_{t}=d_{2} \Delta v-v+u, & x \in \Omega, t>0 \\ \frac{\partial u}{\partial v}=0, \frac{\partial v}{\partial v}=0, & x \in \partial \Omega, t>0 \\ u(x, 0)=u_{0}(x), v(x, 0)=v_{0}(x), & x \in \Omega\end{cases}
$$

in a bounded domain $\Omega \subset \mathbb{R}^{n}(n \geq 1)$ with smooth boundary, where $d_{1}>0$ and $d_{2}>0$ are diffusion coefficients of cell density and chemical stimulus, respectively. The Keller-Segel systems were introduced to describe the aggregation of cellular slime molds, $u$ represents the density of the cells and $v$ represents the concentration of a chemical substance secreted by themselves. The chemical substance is an attractant, they sense a gradient of the chemical substances and move towards higher concentrations. The function $\chi$ is called a sensitivity function, and expresses the relation between the chemical concentration and the cells response, the symbol $\frac{\partial}{\partial v}$ denotes differentiation with respects to the outward normal $v$ on $\partial \Omega$ and the initial data $u_{0}$ and $v_{0}$ are sufficiently smooth functions. For system (1.2) with $d_{1}=d_{2}$, the global existence and boundedness of classical solution is proved under the assumption $0<\chi<\sqrt{\frac{2}{n}}$ see [3] [4] [5]. Lankeit [6] extended the range of $\chi$ in the two-dimensional case. Also the generalized solutions with large $\chi$ are constructed in [3] [7] [8]. More results on the related model with general sensitivity can be found in [9] [10] [11] [12]. In this present paper, we prove the existence of global bounded classical solutions for (1.2) without assumptions on the space dimensions or the smallness assumption on the initial data in the case $d_{1}=d_{2}+\chi$. Our main result reads as follows.

\section{Preliminaries}

Lemma 1.2. (Poincaré inequality) [13] Let $\Omega \subset \mathbb{R}^{n}$ be a bounded domain, then there is exists a constant $C=C(n, p, \Omega)$, such that for all $u \in W^{1, p}(\Omega)$

1) $\|u\|_{W^{1, p}(\Omega)} \leq C\left(\|\nabla u\|_{L^{p}(\Omega)}+\|u\|_{L^{q}(\Omega)}\right), \quad \forall p>1, q>0$.

2) $\left\|u-\frac{1}{|\Omega|} \int_{\Omega} u(x) \mathrm{d} x\right\|_{L^{p}(\Omega)} \leq C\|\nabla u\|_{L^{p}(\Omega)}, \quad \forall 1 \leq p \leq+\infty$.

Theorem 1.1. Let $\Omega \subset \mathbb{R}^{n}(n \geq 1)$ be a bounded domain with smooth boundary and let the parameters $d_{1}>0, d_{2}>0$ and $\chi \in \mathbb{R}$ satisfy $d_{1}=d_{2}+\chi$. Then for any nonnegative function $u_{0} \in C^{0}(\bar{\Omega})$ and positive function $v_{0} \in W^{1, \infty}(\Omega)$, the problem (1.2) has a unique global classical solution which is bounded in $\Omega \times(0, \infty)$.

\section{Proof of Theorem 1.1}

As a preparation to the proof, we first state one result concerning local-in-time classical solution of the problem (1.2), which can be proved by standard contrac- 
tion mapping arguments and parabolic regularity theory (see ([11], Proposition 2.2) and the references therein).

Lemma 3.1. Suppose that $u_{0} \in C^{0}(\bar{\Omega})$ is a nonnegative function and that $v_{0} \in W^{1, \infty}(\Omega)$ is a positive function in $\bar{\Omega}$. Then there exist the maximal existence time $T_{\max } \leq \infty$ and a uniquely determined pair $(u, v)$ of positive functions

$$
\begin{gathered}
u \in C^{0}\left(\bar{\Omega} \times\left[0, T_{\max }\right)\right) \cap C^{2,1}\left(\bar{\Omega} \times\left(0, T_{\max }\right)\right), \\
v \in C^{0}\left(\bar{\Omega} \times\left[0, T_{\max }\right)\right) \cap C^{2,1}\left(\bar{\Omega} \times\left(0, T_{\max }\right)\right) \cap L_{\log }^{\infty}\left(\left[0, T_{\max }\right) ; W^{1, \infty}(\Omega)\right)
\end{gathered}
$$

that solves (1.2) classically in $\Omega \times\left[0, T_{\max }\right)$. In additions, for the second component $v$ of the solution one can find $\eta>0$ such that

$$
\inf _{x \in \Omega} v(x, t) \geq \eta \text { for all } t \in\left(0, T_{\max }\right)
$$

Furthermore, if $T_{\max }<\infty$, Then

$$
\|u(\cdot, t)\|_{L^{\infty}(\Omega)}+\|v(\cdot, t)\|_{W^{1, q}(\Omega)} \rightarrow \infty \text { as } t \nearrow T_{\max } .
$$

The following lemma is a generalization of the maximum principle, which plays a major role in the proof of the main result.

Lemma 3.2. Suppose that $\Omega \subset \mathbb{R}^{n}(n \geq 1)$ is a bounded domain with smooth boundary, $d>0$ is a positive constant and is a positive continuous function satisfying $\int_{0}^{\infty} a(t) \mathrm{d} t<\infty$. Let $z \in C^{0}(\bar{\Omega} \times[0, \infty)) \cap C^{2,1}(\bar{\Omega} \times(0, \infty)), \quad z \geq 0 \quad$ in $\bar{\Omega} \times[0, \infty)$. If

$$
\begin{cases}z_{t} \leq d \Delta z+a(t) z, & x \in \Omega, t>0 \\ \frac{\partial z}{\partial v}=0, & x \in \partial \Omega, t>0 \\ z(x, 0)=z_{0}(x), & x \in \Omega\end{cases}
$$

then $z$ is bounded in $\Omega \times(0, \infty)$.

Proof. Set

$$
y(t):=\max _{x \in \bar{\Omega}} z_{0}(x) \cdot e^{\int_{0}^{t} a(s) d s} \text { for all } t \geq 0 .
$$

By simple calculations we can show that $y$ is the solution of

$$
\left\{\begin{array}{l}
y^{\prime}=a(t) y(t), \quad t>0, \\
y(0):=\max _{x \in \bar{\Omega}} z_{0}(x),
\end{array}\right.
$$

and it is bounded in $(0, \infty)$ by our supposition. Therefore, by the comparison principle, we see that $z$ is bounded in $\Omega \times(0, \infty)$.

We are now in the position to prove global boundedness of solutions for (1.2).

\section{Proof of the Main Result}

Motivated by [14], let us introduce the function $w=\frac{u}{v}$. by using this assumption $d_{1}=\chi+d_{2}$, we shall transform the system (1.2) into 


$$
\begin{cases}w_{1}=d_{1} \Delta w+\frac{2 d_{1}-\chi}{v} \Delta v \cdot \Delta w+(1-w) w, & (x, t) \in \Omega \times\left(0, T_{\max }\right), \\ v_{t}=d_{2} \Delta v-v+v w, & (x, t) \in \Omega \times\left(0, T_{\max }\right), \\ \frac{\partial w}{\partial v}=0, \frac{\partial v}{\partial v}=0, & (x, t) \in \partial \Omega \times\left(0, T_{\max }\right), \\ w(x, 0)=\frac{u_{0}(x)}{v_{0}(x)}, v(x, 0)=v_{0}(x), & x \in \Omega\end{cases}
$$

and then, by the comparison principle we will obtain

$$
w(x, t) \leq \frac{y_{0} \mathrm{e}^{t}}{y_{0} \mathrm{e}^{t}-y_{0}+1},(x, t) \in \Omega \times\left(0, T_{\max }\right)
$$

where $y_{0}:=\max _{x \in \bar{\Omega}} \frac{u_{0}(x)}{v_{0}(x)}$. Hence, the second equation in (1.5) implies that

$$
v_{t} \leq d_{2} \Delta v+\frac{y_{0}-1}{y_{0} \mathrm{e}^{t}-y_{0}+1} v,(x, t) \in \Omega \times\left(0, T_{\max }\right)
$$

If $y_{0} \leq 1$, we deduce that

$$
v(x, t) \leq \max _{x \in \bar{\Omega}} v_{0} x,(x, t) \in \Omega \times\left(0, T_{\max }\right)
$$

by using the maximum principle. For $y_{0}>1$, Let $\bar{v}=\mathrm{e}^{-\left(y_{0}-1\right) t} v$. Through direct computation we establish that

$$
\bar{v}_{t} \leq d_{2} \Delta \bar{v}-\frac{y_{0}\left(y_{0}-1\right)\left(\mathrm{e}^{t}-1\right)}{y_{0} \mathrm{e}^{t}-y_{0}+1} \bar{v}, \quad(x, t) \in \Omega \times\left(0, T_{\max }\right) .
$$

We shall also use the maximum principle for the second time, it follows that

$$
\bar{v}(x, t) \leq \max _{x \in \bar{\Omega}} v_{0} x, \quad(x, t) \in \Omega \times\left(0, T_{\max }\right),
$$

which implies that

$$
v(x, t) \leq \mathrm{e}^{\left(y_{0}-1\right)} \max _{x \in \bar{\Omega}} v_{0} x, \quad(x, t) \in \Omega \times\left(0, T_{\max }\right) .
$$

Along with this, the Lemma 3.1 guarantees that $v(x, t)$ is global in time. Then the integral

$$
\int_{0}^{\infty} \frac{y_{0}-1}{y_{0} \mathrm{e}^{t}-y_{0}+1} \mathrm{~d} t<\infty,
$$

we apply the Lemma 3.2 to (1.6), it follows that $v$ is bounded in $\Omega \times(0, \infty)$, and hence $u=v w$ is bounded in $\Omega \times(0, \infty)$ with smooth boundary, $\Omega \subset \mathbb{R}^{n}(n \geq 1)$, Thus we complete the proof.

\section{Conclusion and Remarks}

In the paper, we presented that the Neumann initial-boundary value problem for the chemotaxis system with singular sensitivity in problem (0.1) is bounded in $\Omega \times(0, \infty)$ with smooth boundary, $\Omega \subset \mathbb{R}^{n}(n \geq 1)$. Then we established that the problem (1.2) has a unique global classical solution which is bounded in $\Omega \times(0, \infty)$. And we showed that $\Omega \subset \mathbb{R}^{n}(n \geq 1)$ is a bounded domain with smooth boundary, $d>0$ is a positive constant and a is a positive continuous 
function.

\section{Acknowledgements}

The authors would like to thank the anonymous referees for their helpful comments. Referees' comments led to improvements of this paper.

\section{Conflicts of Interest}

The authors declare no conflicts of interest regarding the publication of this paper.

\section{References}

[1] Keller, E.F. and Segel, L.A. (1970) Initiation of Slime Mold Aggregation Viewed as an Instability. Journal of Theoretical Biology, 26, 399-415. https://doi.org/10.1016/0022-5193(70)90092-5

[2] Bellomo, N., Bellouquid, A., Tao, Y. and Winkler, M. (2015) Toward a Mathematical Theory of Keller-Segel Models of Pattern Formation in Biological Tissues. Mathematical Models and Methods in Applied Sciences, 25, 1663-1763. https://doi.org/10.1142/S021820251550044X

[3] Winkler, M. (2011) Global Solutions in a Fully Parabolic Chemotaxis System with Singular Sensitivity. Mathematical Methods in the Applied Sciences, 34, 176-190. https://doi.org/10.1002/mma.1346

[4] Fujie, K. (2015) Boundedness in a Fully Parabolic Chemotaxis System with Singular Sensitivity. Journal of Mathematical Analysis and Applications, 424, 675-684. https://doi.org/10.1016/j.jmaa.2014.11.045

[5] Winkler, M. and Yokota, T. (2018) Stabilization in the Logarithmic Keller-Segel System. Nonlinear Analysis, 170, 123-141. https://doi.org/10.1016/j.na.2018.01.002

[6] Lankeit, J. (2016) A New Approach toward Boundedness in a Two-Dimensional Parabolic Chemotaxis System with Singular Sensitivity. Mathematical Methods in the Applied Sciences, 39, 394-404. https://doi.org/10.1002/mma.3489

[7] Stinner, C. and Winkler, M. (2011) Global Weak Solutions in a Chemotaxis System with Large Singular Sensitivity. Nonlinear Analysis: Real World Applications, 12, 3727-3740. https://doi.org/10.1016/j.nonrwa.2011.07.006

[8] Lankeit, J. and Winkler, M. (2017) A Generalized Solution Concept for the Keller-Segel System with Logarithmic Sensitivity: Global Solvability for Large Nonradial Data. Nonlinear Differential Equations and Applications NoDEA, 24, Article number: 49. https://doi.org/10.1007/s00030-017-0472-8

[9] Mizukami, M. and Yokota, T. (2017) A Unified Method for Boundedness in Fully Parabolic Chemotaxis Systems with Signal-Dependent Sensitivity. Mathematische Nachrichten, 290, 2648-2660. https://doi.org/10.1002/mana.201600399

[10] Black, T., Lankeit, J. and Mizukami, M. (2019) Stabilization in the Keller-Segel System with Signal-Dependent Sensitivity. Applicable Analysis. https://doi.org/10.1080/00036811.2019.1585534

[11] Fujie, K. and Senba, T. (2016) Global Existence and Boundedness of Radial Solutions to a Two Dimensional Fully Parabolic Chemotaxis System with General Sensitivity. Nonlinearity, 29, 2417-2450. https://doi.org/10.1088/0951-7715/29/8/2417

[12] Fujie, K. and Senba, T. (2018) A Sufficient Condition of Sensitivity Functions for 
Boundedness of Solutions to a Parabolic-Parabolic Chemotaxis System. Nonlinearity, 31, 1639-1672. https://doi.org/10.1088/1361-6544/aaa2df

[13] Horstmann, D. and Winkler, M. (2005) Boundedness vs. Blow-Up in a Chemotaxis System. Journal of Differential Equations, 215, 52-107. https://doi.org/10.1016/j.jde.2004.10.022

[14] Yang, Y., Chen, H. and Liu, W. (2001) On Existence of Global Solutions and Blow-Up to a System of the Reaction-Diffusion Equations Modelling Chemotaxis. SIAM Journal on Mathematical Analysis, 33, 763-785.

https://doi.org/10.1137/S0036141000337796 